\title{
Literaturbericht
}

Dorothea Prell

\section{Politische Psychologie als Perspektive und Potential der politikwissenschaftlichen Analyse}

\section{Einleitung}

Die Politische Psychologie (PP) ist sowohl in Deutschland als auch im angloamerikanischen Raum ein relativ junger, interdisziplinär veranlagter Forschungszweig, der sich seit einigen Jahren eines verstärkten wissenschaftlichen Interesses erfreut und sich unter anderem Fragen widmet, die auf die Persönlichkeit von politischen Entscheidungsträgern oder auf die Performanz von politischen Gruppen abzielen: Warum verhalten sich Politiker in einer bestimmten Weise? Wie treffen sie ihre politischen Entscheidungen? Worin liegen die Ursachen von ethnischen Konflikten? Unter welchen Bedingungen ist eine Versöhnung in Post-Konflikt-Situationen möglich? Dies sind nur einige der Fragen, für die die PP durch die Analyse von kognitiven Prozessen, Emotionen und Motivationen von Individuen und Gruppen versucht, Erklärungsansätze zu finden und Antworten zu geben.

Obwohl die Politische Psychologie europäische Wurzeln besitzt, hat sie sich insbesondere im angloamerikanischen Raum entwickelt und dort eindrucksvoll ausdifferenziert (Jost/Sidanius 2004 a: 2). Dennoch ist sie international ausgerichtet und strahlt in zunehmenden Maße auch bis nach Europa und Australien aus (Houghton 2009: 23 f.). In Deutschland blickt die PP auf eine ambivalente Vorgeschichte zurück, da sie einerseits unter den Bedingungen des Nationalsozialismus als Instrument der faschistischen Massenmanipulation eingesetzt wurde, andererseits aber zugleich eine kritische Reaktion (Adorno/Frenkel-Brunswik/Levinson 1950) und Aufarbeitung dieser Entwicklungen ermöglichte (Wasmuth 2011: 471). Infolgedessen war die PP in Deutschland nach 1945 lange ein Tabu-Thema oder allenfalls eine „Randerscheinung“ (Preiser 1993: 522). Seit 1958 ist sie im Berufsverband Deutscher Psychologinnen und Psychologen (BDP) in einer eigenen Sektion orga- 
nisiert. ${ }^{1}$ Auch in den akademischen Strukturen der Politikwissenschaft ist die PP vertreten. So existiert in der Deutschen Vereinigung für Politische Wissenschaft (DVPW) ein Arbeitskreis „Politische Psychologie“, der eng mit dem SigmundFreud-Institut (SFI) in Frankfurt/Main verbunden ist. ${ }^{2}$

Trotz dieser organisatorischen Einbindung fristet die PP in Deutschland bedauerlicherweise ein Schattendasein (Frick 2007: 9). Anders als in den USA gehört sie bisher weder zum festen politikwissenschaftlichen Repertoire an deutschen Hochschulen, noch existiert ein Lehrstuhl oder eine Professur an politikwissenschaftlichen Instituten, die sich hauptsächlich mit Themen der Politischen Psychologie in Lehre und Forschung auseinandersetzt. ${ }^{3}$ In der Politikwissenschaft und auch in der Psychologie (Sozialpsychologie) beschäftigt man sich vorzugsweise mit Subfeldern der PP, wie beispielsweise der Wahl- und Einstellungsforschung (Gabriel/Wessels/ Falter 2009) oder der politischen Kulturforschung (Westle/Gabriel 2009). Diesem Umstand ist es wohl geschuldet, dass ein Großteil der aktuellen Forschung überwiegend aus den USA stammt. Zudem ist der amerikanische Themenkanon, wie im Folgenden noch gezeigt wird, sehr vielseitig und stark international ausgerichtet. Die amerikanische PP widmet sich daher nicht nur national begrenzten Untersuchungsgegenständen, sondern auch globalen Fragestellungen aus dem Gebiet der Internationalen Beziehungen. Mit dieser Themenvielfalt ist sowohl eine Fülle an grundlegenden als auch an aktuellen, problemorientierten Publikationen verbunden.

Vor diesem Hintergrund will der folgende Literaturbericht das Feld der PP überblicksartig darstellen und auf diesem Wege Perspektiven und Potentiale der PP skizzieren. Der Schwerpunkt des Literaturberichtes liegt dabei auf angloamerikanischen Beiträgen. Dazu wird im Folgenden zunächst geklärt, was unter Politischer Psychologie zu verstehen ist und welches ihre wichtigsten Untersuchungsgegenstände sind. Exemplarisch wird dabei der Themenschwerpunkt Politik und Persönlichkeit detaillierter betrachtet. Abschließend wird der Frage nachgegangen, worin der Mehrwert und die Anschlussfähigkeit politisch-psychologischer Zugänge für politikwissenschaftliche Fragestellungen bestehen.

1 Vergleiche die Seite der Sektion Politische Psychologie des BDP http://www.bdp-politische.de/ (Stand: 30.10.2011).

2 Vergleiche die Seiten der DVPW https://www.dvpw.de/nc/gliederung/ak.html und des SFI http:// www.sfi-frankfurt.de/home.html (Stand: 30.10.2011).

3 Dieser Umstand wird auch durch das Vorhaben der Sektion „Politische Psychologie” der BDP bestätigt, die derzeit das aktuelle Lehrangebot zum Thema PP an deutschen Hochschulen evaluiert und damit das Ziel verfolgt, ein ähnlich strukturiertes Ausbildungsangebot zu entwickeln, wie man es auch an Hochschulen im angloamerikanischen Sprachraum vorfinden kann. Vergleiche die Anfrage der Sektion "Politische Psychologie" BDP vom 25.10.2011. 
Politische Psychologie

\section{Was versteht man unter Politischer Psychologie?}

\section{a) Begriffsbestimmung}

Für ein erstes, allgemeines Verständnis kann zunächst die Definition von Sears, Huddy und Jervis herangezogen werden, die unter PP die Anwendung psychologischer Erkenntnisse und Konzepte für die politikwissenschaftliche Analyse verstehen: „Political psychology is, at the most general level, an application of what is known about human psychology to the study of politics." (2003 a: 3). PP verfolgt demnach das Ziel, psychologische Ursachen für politisches Verhalten aufzuzeigen und dieses zu erklären. Dieser Lesart folgend, wonach der Fokus der PP auf der Erklärbarkeit politischer Phänomene liegt, erläutern auch Cottam u. a.: „Political Psychology involves explaining what people do, by adapting psychological concepts, so that they are useful and relevant to politics, then applying them to the analysis of a politcal problem or issue" (2010 a: 4). Deutsch und Kinnvall hingegen legen in ihrem viel beachteten Aufsatz „What Is Political Psychology?“ den Schwerpunkt auf die Interaktion politischer und psychologischer Prozesse: ,the field of political psychology is the study of the interaction of political and psychological processes; this is a bidirectional interaction. Just as cognitive capabilities limit and affect the nature of the political and social world of political agents so, also, the structures and processes of politics affect cognitive capabilities" (2002: 17). Auch Jost und Sidanius heben in ihrer Begriffsbestimmung die Wechselwirkung zwischen politischen Institutionen und menschlichem Verhalten hervor: „Political Psychology explores the border that runs between the intellectual nations of political science and psychology. It is a dynamic subfield that adresses the ways in which political institutions both affect and are affected by human behavior" (2004 a: 1). ${ }^{4}$

Dieser kurze Blick auf die Vielfalt der Begriffsbestimmungen zeigt, dass eine einheitliche und allgemeingültige Definition von Politischer Psychologie nicht existiert. Dies ist unter anderem der ausgeprägten Interdisziplinarität geschuldet. So finden nicht nur theoretische Ansätze und Methoden aus der Politikwissenschaft und der Psychologie Anwendung. Es bestehen auch Überschneidungen mit anderen Disziplinen wie der Geschichte, Soziologie, Anthropologie, Pädagogik oder Kommunikationswissenschaft, die wiederum eine Vielzahl von Perspektiven auf die PP ermöglichen und keine definitorische Verengung zulassen (Hermann 1986: 2; Houghton 2009: 22 f.; Jost/Sidanius 2004 a: 1).

Wohl aber lassen sich gemeinsame Grundsätze ausmachen, welche die Mehrzahl der Autoren innerhalb der PP als wesentliche Bestimmungsfaktoren anerkennen und

4 Vergleiche dazu auch Bleek 2001. 
die als Orientierungspunkte in der Beschäftigung mit dieser dienen sollen. Hermann, eine der führenden Wegbereiter der PP in den USA, formulierte in ihrem 1986 veröffentlichten Handbuch „Political Psychology“ erstmals fünf Grundsätze der PP, auf deren Grundlage sich die Debatte bis heute kontinuierlich herausgebildet hat. Der erste besagt, dass der Fokus auf der Interaktion zwischen politischen und psychologischen Phänomenen liegt. Dies bedeutet, dass Wahrnehmungen, Überzeugungen, Motivationen und Werte das politische Verhalten von Akteuren beeinflussen und diese zugleich durch kulturelle und strukturelle Faktoren des politischen Systems geprägt werden. ${ }^{5}$ Der zweite Grundsatz lautet, dass die Forschung auf gesellschaftiche Probleme reagiert und für diese relevant ist. Hermann zufolge kommt eine Vielzahl der interessierten Wissenschaftler mit PP in Berührung, weil sie nach Antworten auf drängende gesellschaftliche Probleme oder Herausforderungen wie Umweltprobleme, nukleare Bedrohung, Ursachen für Gewalt und Konflikte etc. suchen. Zur Bearbeitung dieser Herausforderungen ist gerade die Analyse der Interaktion von Individuen, Gruppen und Politik entscheidend. Der dritte Grundsatz unterstreicht, dass die Berücksichtigung des Kontextes einen Unterschied machen kann. Hiermit ist gemeint, das Individuum zu einer bestimmten Zeit und in einer spezifischen Situation, das heißt innerhalb eines bestimmten politischen Systems und einer politischen Kultur, zu erforschen. Die Berücksichtigung dieser Kontextfaktoren im Einfluss auf und in der Interaktion mit dem Individuum trägt in der Analyse entscheidend dazu bei, Aussagen zur Persönlichkeit eines Individuums und zu seinem politischen Einfluss in einer bestimmten Situation zu treffen. Der vierte Grundsatz besagt, dass der Schwerpunkt sowohl auf dem Prozess als auch auf dem Ergebnis liegt. Für die PP sind demnach sowohl die Herausbildung politischen Verhaltens (Prozess) als auch das politische Verhalten an sich (Ergebnis) von Bedeutung. Der fünfte Grundsatz bringt schließlich zum Ausdruck, dass in der Datenerhebung multiple Methoden zur Anwendung kommen sollen. Dies bedeutet, dass kein methodischer Dogmatismus, sondern Offenheit gegenüber diversen Methoden der involvierten Disziplinen herrscht. So werden in Abhängigkeit vom Untersuchungsgegenstand Inhaltsanalysen, Experimente, Diskursanalysen, Umfragen, Interviews und Fallstudien durchgeführt oder auch Fokusgruppen eingesetzt (Hermann 1986: 1-4).

Diese Grundsätze wurden in der weiteren Entwicklung sowohl von Hermann als auch von anderen Autoren (Hermann 1986, 2002 a, 2004 a; Deutsch/Kinnvall 2002; McDermott 2004) in zahlreichen Veröffentlichungen aufgegriffen, wiederholt eva-

5 Vergleiche dazu auch in der deutschsprachigen Literatur Mayer/Kortmann 2010 sowie Pickel/Pickel 2006. 
luiert und teils neu akzentuiert (etwa mit Blick auf das Individuum als Untersuchungseinheit der PP; Hermann 2004 a: 12-16). Gleichwohl bleiben sie für die weitere Auseinandersetzung mit PP richtungsweisend (Deutsch/Kinnvall 2002: $33 \mathrm{ff}$.). Unter Berücksichtigung dieser Orientierungspunkte in der politikwissenschaftlichen Analyse kann die Politische Psychologie als alternativer Zugang oder ebenso als Perspektive auf unterschiedliche Fragestellungen und Phänomene im Bereich der Politikwissenschaft dienen.

\section{b) Etappen und Entstehungsgeschichte der Politischen Psychologie}

Jost und Sidanius sowie Ward verweisen auf die lange Tradition der Politischen Psychologie innerhalb der europäischen politischen Ideengeschichte. Da die PP Fragen nach der Natur des Menschen und nach der Konstitution des politischen Gemeinwesens berührt, können die Ursprünge bereits im „Il Principe“ Machiavellis oder in den Vertragstheorien Hobbes', Rousseaus und Lockes gefunden werden (ausführlich Ward 2002; Jost/Sidanius 2004 a). Deutsch und Kinnvall verorten die Anfänge der PP dagegen in der Zwischenkriegszeit. Das Entstehen, das grausame Wirken und die Zerstörungskraft totalitärer Regime, das Aufkommen der Massenmedien und deren gezielter Einsatz für die Propaganda machten schließlich systematische Erkenntnisse zum Zusammenhang zwischen psychologischen und politischen Prozessen erforderlich (Deutsch/Kinnvall 2002: 15).

In der ersten Hälfte des 20. Jahrhunderts bildete sich die PP als akademische Disziplin in den USA heraus. Als einer der ersten Politikwissenschaftler setzte sich Merriam für die Zusammenführung von Politikwissenschaft und Psychologie ein. Sein Schüler Lasswell war in der Umsetzung dieses Vorhabens jedoch viel erfolgreicher als sein Mentor und gilt daher als Vater der amerikanischen Politischen Psychologie. Sein wissenschaftlicher Ansatz beruhte auf den psychoanalytischen Erkenntnissen Freuds und konzentrierte sich auf den Einfluss psychologischer Prozesse auf politische Ergebnisse. Eine seiner Hauptthesen besagt, dass Menschen ihre ungelösten und nicht erkannten psychischen Konflikte auf die externe politische Welt projizieren (McDermott 2004: 4). ${ }^{6}$

In den 1970er Jahren hatte sich die PP in den USA vollends etabliert. Einen wesentlichen Meilenstein stellte 1973 die Veröffentlichung des ersten umfassenden und international anerkannten „Handbook of Political Psychology“ dar. Dieses von Knutson herausgegebene Einführungswerk war für die Disziplin wegweisend und

6 Zu Lasswells bedeutendsten Werken zählen „Psychopathology and Politics“(1930), „World Politics and Personal Insecurity“ (1935), „Politics: Who Gets What, When and How“ (1936) und „Power and Personality“ (1948). 
enthielt Beiträge führender Wissenschaftler, die den zu jener Zeit aktuellsten Stand zur Forschung und zur Entstehungsgeschichte der PP abbildeten. Mit der Gründung der „International Society of Political Psychology“ (ISPP) im Jahr 1978 hatte sich die Politische Psychologie auch institutionell gefestigt. Seit 1979 bringt die ISPP vierteljährlich das renommierte, interdisziplinäre ,Journal of Political Psychology“ heraus. 1986 erschien das bereits zitierte Handbuch "Political Psychology" von Hermann, das sich an der Struktur des Handbuchs von Knutson orientierte und den aktuellen Stand der PP in Wissenschaft und Forschung zu dieser Zeit dokumentierte. Dieser Tradition folgend gab die Politikwissenschaftlerin Monroe 2002 ein drittes Handbuch „Political Psychology“ heraus. Auch Monroe und Kollegen zeichnen ein aktuelles Bild der PP im 21. Jahrhundert, indem sie einen Überblick über das Forschungsgebiet geben, spezielle Bereiche der PP betrachten und künftige Forschungsschwerpunkte identifizieren (Monroe 2002 a). Bereits im darauf folgenden Jahr erschien das von Sears, Huddy und Jervis herausgebene „Oxford Handbook of Political Psychology". Dieses unterscheidet sich von den bereits vorgestellten Handbüchern, da es sich hier um ein Nachschlagewerk handelt, das sich in fünf Teile - Theoretische Ansätze, Internationale Beziehungen, politisches Verhalten der Masse, Intergruppenbeziehungen und politischer Wandel - gliedert und in Überblicksaufsätzen zentrale Themen der PP vorstellt (Sears/Huddy/Jervis 2003 b). Die ISPP begründete 2004 schließlich die Serie „Advances in Political Psychology“, deren erster Band im gleichen Jahr von Hermann herausgegeben wurde. Hier erörtert sie den aktuellen Stand der Forschung der PP. Zudem werden in sechs Beiträgen die neuesten Erkenntnisse zu Themen wie Identität, Entscheidungsfindung oder politischer Partizipation, die auf Grundlage verschiedenster Methoden zustande gekommen sind, dargestellt (Hermann 2004 b).

Für die Entwicklung der Politischen Psychologie kann abschließend festgehalten werden, dass die in regelmäßigen Abständen erschienenen Handbücher einerseits als Antwort auf dieses sich rasant ausdifferenzierende und anwachsende Forschungsgebiet verstanden werden können, diese andererseits aber auch eine konstitutive Funktion in ihrem Entwicklungsprozess erfüllt haben.

\section{Themengebiete der Politischen Psychologie}

Ein großes thematisches Spektrum gehört zu den auffallenden Charakteristika der Politischen Psychologie. Die Themen reichen von Wähler- und Gruppenverhalten, Emotionen und Kognition über politische Führung, ethnische Konflikte, Genozid 
oder politischen Extremismus bis hin zu Terrorismus, Krieg und Abschreckung (Cottam u. a. 2010 a: 9). ${ }^{7}$

Der Sozialpsychologe McGuire analysiert in seinem vielzitierten Aufsatz „The Poly-Psy Relationship“ (2004) das Verhältnis zwischen den Disziplinen Politikwissenschaft und Psychologie. Er systematisiert die intellektuelle Entwicklung der PP und teilt diese chronologisch in drei Phasen ein. Für jede dieser Phasen identifiziert er einen thematischen Fokus und ordnet diesem Theorien und Methoden zu, die in den jeweiligen Phasen en vogue waren. Diese Phasen stecken drei große Themenkomplexe ab, die im Zuge des wissenschaftlichen Fortschritts weiter ausdifferenziert wurden und zu denen bis heute kontinuierlich geforscht wird.

In der 1. Phase Persönlichkeit und Kultur (1940er und 1950er Jahre) bestand die Forschungsmotivation darin, politisches Denken, Fühlen und Handeln zu erklären. Unter Zuhilfenahme von Konzepten der Psychoanalyse, des Behaviorismus und des Marxismus wurde die Frage diskutiert, ob situative oder dispositive Faktoren menschliches Handeln determinieren (McGuire 2004: 23). In dieser Analyse standen Persönlichkeitsstudien politischer Führungsfiguren im Vordergrund, deren Persönlichkeit und Führungsstil auf Grundlage der damals sehr einflussreichen Psychoanalyse Freuds in Form von Psychobiografien analysiert wurden (zum Beispiel George/George 1956; Erikson 1958). Mit dem Fortschreiten der Forschung rückten zunehmend Persönlichkeitsanalysen in das Zentrum des Interesses, die Charaktereigenschaften und Motivationen politischer Führungsfiguren in ihre Untersuchung mit einbezogen (Cottam u. a. 2010 a: 6).

Die 2. Phase Politische Einstellung und Wahlverhalten (1960er und 1970er Jahre) begann mit der systematischen Erforschung der öffentlichen Meinung, der politischen Einstellung und des Wahlverhaltens in den USA (ebd.). In der Einstellungsforschung wurde insbesondere zum Zusammenhang von Einstellungen und Verhalten gearbeitet. Geraten Einstellung und tatsächliches Verhalten in Widerspruch, erlebt das Individuum psychologische Spannungen, die es abbauen möchte (Houghton 2009: 28). Diesen Zustand der kognitiven Dissonanz und wie Individuen mit diesem Phänomen der Inkonsistenz umgehen, beschreibt Festinger in seiner ,Theory of Cognitive Dissonance" (1957). Im Zuge der kritischen Auseinandersetzung mit den Konsistenztheorien wendete man sich später neueren Ansätzen wie der Attributionstheorie (Jones u. a. 1972) oder der Schematheorie (Kuklinski/Luskin/Bolland 1991) zu.

7 Vergleiche Knutson 1973; Hermann 1986; Monroe 2002 b; Sears/Huddy/Jervis 2003 b; Jost/Sidanius 2004 b; Houghton 2009; Cottam u. a. 2010 b. 
Für die Wahlforschung war die Veröffentlichung des Werkes „The American Voter" im Jahr 1960 wegweisend. Campbell u. a. kommen hier zu dem Schluss, dass subjektive, psychologische Faktoren, letztlich die Parteiidentifikation, für eine Wahlentscheidung ausschlaggebend sind. Für die Analyse des Wahlverhaltens wurde zudem die Rational Choice-Theorie angewendet, die - stark verkürzt dargestellt - von der Annahme ausgeht, dass Menschen in ihrem Handeln von ihrem Eigeninteresse geleitet werden und dabei ein Kosten-Nutzen-Kalkül verfolgen (McGuire 2004: 27). $\mathrm{Zu}$ den bedeutendsten Werken der Wahlforschung, die auf diesem rationalen Ansatz beruhen, zählt Downs' „An Economic Theory of Democracy“ (1957).

In der 3. Phase Politische Überzeugungen, Informationsverarbeitung und Entscheidungsfindung (1980er und 1990er Jahre) richtete sich der Fokus auf den Inhalt und die Wirkungsweise des kognitiven Systems während des Prozesses der Entscheidungsfindung in der politischen Sphäre. Als Beispiel für die Analyse politischer Überzeugungen bzw. Glaubenssysteme sowie der Entscheidungsfindung seien etwa das Analyseraster des ,operational code“ (George 1971) und die Methode der „,cognitive maps“ (Axelrod 1976) erwähnt. Zahlreiche weitere Ansätze im Bereich der Kognition und Entscheidungsfindung gehen kurzgefasst davon aus, dass Individuen begrenzte kognitive Kapazitäten besitzen und eine Reihe von mentalen „short-cuts“, darunter Heuristiken und Analogien, nutzen, um sich in der komplexen Umwelt orientieren und Entscheidungen treffen zu können (McGuire 2004: $29 \mathrm{f}$., Houghton 2009: 30). Die Ansätze der dritten Phase kamen insbesondere im Bereich der Internationalen Beziehungen zum Einsatz (Mandel 1986; Hermann 1980 b, 1993, 2001; Hermann/Hagan 1998; Renshon/Renshon 2008).

Darüber hinaus gibt McGuire einen Ausblick auf eine 4. Phase, in der sich ein Trend von der Beschäftigung mit intrapersonellen Themen (Persönlichkeit, Einstellung, Kognition) hin zur Beschäftigung mit interpersonellen und Intergruppenbeziehungen innerhalb der Internationalen Beziehungen beobachten lässt (McGuire 2004: 31). Dies wird durch jüngere Publikationen zu Gewalt und Konflikt in den Internationalen Beziehungen (Gross Stein 2008), zu Nationalismus, ethnischen Konflikten (Volkan 1999), Genozid (Baum 2008; Staub 2000, 2006; Chirot/McCauley 2010) und Terrorismus (Crenshaw 2004, 2007; Post 2005 a, 2009) etc. belegt.

Neue Ansätze der Politischen Psychologie zum Verständnis politischer Entscheidungsprozesse kommen seit einigen Jahren aus den Neurowissenschaften. Mittels funktioneller Magnetresonanztomographie (fMRT) kann die Hirnaktivität durch den höheren Sauerstoffgehalt in aktiven Hirnregionen sichtbar gemacht werden. In einer Studie während der Präsidentschaftswahlen 2004 in den USA wurden demo- 
kratischen und republikanischen Wählern die Bilder der drei Präsidentschaftskandidaten Bush, Kerry und Nader gezeigt und dabei die Hirnaktivität mittels fMRT gemessen (Kaplan/Freedman/Iacoboni 2007). Die Betrachtung von Kandidaten nicht favorisierter Parteien führte nicht nur zur Aktivierung emotionaler Regionen bei den Probanden, sondern auch zur Aktivierung kognitiver Bereiche. Ähnlich wie bei der Betrachtung von Gesichtern unterschiedlicher Ethnien (Cunningham u. a. 2004; Richeson u. a. 2003) oder von Angst auslösenden Situationen (Johanson u. a. 1998) dient die Aktivierung dieser kognitiven Areale möglicherweise der Unterdrückung und Kontrolle von negativen Emotionen (Dolcos/Iordan/Dolcos 2011). Unterschiedliche Aktivierungsmuster fanden sich auch in Abhängigkeit von verschiedenen politischen Überzeugungen (Zamboni u. a. 2009; Westen u. a. 2006). Die Hirnstrukturen, die beispielsweise bei konservativen oder radikalen Einstellungen vermehrt aktiviert zu sein scheinen, spielen auch bei generellen kognitiven Prozessen wie der ,self-other"-Prozessierung oder der sozialen Entscheidungsfindung in ambivalenten Situationen eine Rolle (Zamboni u. a. 2009). Solche neuronalen Korrelate können dazu beitragen, bewusste und unbewusste politische Entscheidungsprozesse und Überzeugungen zu klassifizieren und neu zu beleuchten, doch stellen sich gerade bei diesen Studien Fragen nach den methodischen Grenzen, etwa dem gewählten statistischen Ansatz, der Auswahl der Probanden und Kontrollen, dem Untersuchungssetting ebenso wie die generelle Frage nach dem Nutzen und Mehrwert hinsichtlich der Ausgangsfrage nach dem Verständnis politischer Vorgänge.

Ein weiteres aktuelles und wachsendes Feld der Politischen Psychologie behandelt die Rolle der Emotionen bei politischen Problemen und Prozessen. Die Untersuchungen zu diesem komplexen und multidimensionalen Thema reichen von individuellen Blickwinkeln auf gesellschaftliche Ungleichheit (Montada/Schneider 1989) über die Verarbeitung von Gewalt und Terroranschlägen (Dumont u. a. 2003; Healy u. a. 2009) bis hin zu Emotionen bei Gruppenkonflikten (De Rivera/Páez 2007) und dem Verständnis von Fairness als Grundlage der sozialen Kooperation (Skitka 2002). Insbesondere die relative Deprivation oder das Gefühl der Unzufriedenheit und sozialen Benachteiligung werden als wichtiger Auslöser politischer Aktivität und Protests angesehen (Walker/Smith 2002; Leach 2010).

In diesem Kontext sei noch auf den aktuellen Sammelband „Information - Wahrnehmung - Emotion" von Faas, Arzheimer und Roßteutscher (2010) verwiesen. Schemer, Wirth und Matthes widmen sich hier insbesondere der Bedeutung von Emotionen in politischen Entscheidungsprozessen, indem sie den Einfluss von Emotionen und Affekten auf das Wahlverhalten am Beispiel der Abstimmung über die Asylgesetzverschärfung in der Schweiz untersuchen (2010). Auch werden in 
diesem Sammelband neueste und besonders innovative methodische Ansätze der Politischen Psychologie im Bereich der deutschen Wahl- und Einstellungsforschung vorgestellt. So kam etwa die Technologie des Eye-tracking zur Untersuchung des Blickverlaufs der Rezipienten bei der Wahrnehmung und Bewertung von Wahlplakaten zum Einsatz (Geise/Brettschneider 2010).

\section{Persönlichkeit und Politik}

Wie bereits thematisiert, besitzt die Beschäftigung mit der Persönlichkeit und deren Einfluss auf die Politik eine lange Tradition und stellt den größten thematischen Bereich innerhalb der Politischen Psychologie dar. Im Folgenden werden einige bedeutende Publikationen und Ansätze vorgestellt. ${ }^{8}$

Der Forschungsbereich zu Persönlichkeit und Politik lässt sich nach Winter (2003: 110) zunächst zweiteilen. Der Fokus richtet sich zum einen auf die Forschung zum Massenverhalten, wobei hier unter anderem die interpersonelle Beziehung zwischen politischer Führungsfigur und „Anhängerschaft“, politische Einstellung und Wahlverhalten, oder der Einfluss der öffentlichen Meinung auf Inhalte der Regierungspolitik behandelt wird. Ein zweiter Bereich beschäftigt sich mit der politischen Elite und deren Führungsstilen oder Entscheidungsfindungsprozessen, zum Beispiel in der Außenpolitik. In der Betrachtung der politischen Elite stellen Ansätze und Analyserahmen zu Political Leadership einen Hauptteil der theoretischen Auseinandersetzung dar. Aufgrund ihrer eng miteinander verflochtenen, intellektuellen Entwicklung lassen sich laut Cottam u. a. (2010 a: 13) Ansätze zur Untersuchung von Persönlichkeit kaum mehr von Ansätzen zu Political Leadership trennen. ${ }^{9}$

Beschäftigt man sich mit dem Faktor Persönlichkeit im Bereich der Politischen Psychologie, tauchen eine Reihe von grundlegenden Fragen auf, die bereits bei der Begriffsbestimmung zur PP kurz angesprochen wurden, etwa: Wann bzw. in welchem Kontext können Persönlichkeiten politische Prozesse beeinflussen? Wie kann die politische Performanz eines Akteurs methodisch analysiert oder gemessen werden, wo doch den meisten Wissenschaftlern ein direkter Zugang zu hochrangigen Politikern und deren Machtzirkeln verwehrt bleibt?

8 Einen ausführlichen Überblick und weiterführende Literatur zu einzelnen Ansätzen und spezifischen Analysen liefert Winter in seinem Essay „Personality and Political Behavior“ (2003) sowie Cottam u. a. im zweiten Kapitel „Personality and Politics“ ihres Einführungswerkes „Political Psychology“ (2010b).

9 Als Beispiele der deutschsprachigen Literatur zum Thema Politische Führung vergleiche Fagagnini 2000; Fliegauf/Kießling/Novy 2008; Gast 2007, 2009; Glaab 2010; Grasselt/Korte 2007; Helms 2000, 2005; Jankowitsch 2008; Sebaldt/Gast 2010. 
Als Antwort auf die erste Frage formulierte Greenstein folgende Bedingungen, unter denen der politische Einfluss eines Akteurs wahrscheinlich sein kann: wenn die Umgebung eine Restrukturierung zulässt, wenn sich der politische Akteur in einer strategisch günstigen Position befindet und in Abhängigkeit seiner persönlichen Stärken und Schwächen (Greenstein 1969, 1992). Byman und Pollack ihrerseits sehen einen wahrscheinlichen Einfluss von Individuen in den Internationalen Beziehungen als gegeben, wenn sich die politische Macht in den Händen eines einzelnen politischen Führers konzentriert, wenn sich Institutionen im Konflikt befinden und in Zeiten politischen Wandels (2001: 109). Zudem sei auf ein weiteres grundlegendes Merkmal in der Beschäftigung mit der Persönlichkeit in der Politik hingewiesen: Die Untersuchung des Einflusses von Persönlichkeiten in der Politik, insbesondere in ihrer Entscheidungsfindung und im Umgang mit politischen Krisen, findet zumeist in der außenpolitischen Arena statt, da eine grundlegende Prämisse besagt, dass Akteure in der Regel im Bereich der Außenpolitik einen größeren politischen Spielraum als in der Innenpolitik besitzen (Post/Walker/Winter 2005: 2; Winter 1992).

Auch mit Blick auf die zweite eingangs erwähnte Frage nach praktikablen methodischen Untersuchungsmöglichkeiten, die einen direkten Zugang zu politischen Entscheidungsträgern nicht voraussetzen, lassen sich einige Orientierungspunkte benennen. So hat sich in der Politischen Psychologie etwa die sogenannte assessment-at-a-distance technique etabliert, die es Wissenschaftlern ermöglicht, auch auf Distanz Profile politischer Akteure zu erstellen. In seinem Review-Essay zum Thema „Assessing Leaders' Personalities“ aus dem Jahr 2005 stellt Winter die wichtigsten Entwicklungen in diesem Bereich vor, angefangen bei den verschiedenen Typen der Psychobiographie über einzelne Motiv-Studien (,,Thematic Apperception Test-Based Measures“), „operational codes“ bis hin zu komplexen Methoden, die einen integrativen Ansatz verfolgen, wie die Leadership Trait Analysis.

Mit Blick auf das Spektrum möglicher Theorien lassen sich psychoanalytische Ansätze, Traittheorien und Motivtheorien (Cottam u. a. 2010 a: $13 \mathrm{f}$.) unterscheiden. $\mathrm{Zu}$ den psychoanalytischen Ansätzen gehört zum einen die psychobiographische Methode, die maßgeblich durch die psychoanalytischen Errungenschaften Freuds geprägt und bereits vorab genannt wurde. Eine der wenigen aktuellen Veröffentlichungen in der deutschen Fachliteratur, die einen psychobiographischen Ansatz verfolgt und die Persönlichkeit anhand psychologischer Erklärungsmuster analysiert, stammt von Hartmann. In „Persönlichkeit und Politik“ (2007) untersucht er mit Hilfe eines Kriterienkataloges die Persönlichkeiten von Staats- und Regierungschefs aus Deutschland, Frankreich, Großbritannien, den USA, Russland sowie China und bietet eine umfangreiche Sammlung an Politikerpersönlichkeiten, deren 
individuelle politische Karrieren in ihren jeweiligen historischen und politischen Kontexten betrachtet werden. Weitere Ansätze im Bereich der Psychoanalyse widmen sich der Psychopathologie und dem Spektrum psychologischer Störungen politischer Persönlichkeiten (Cottam u. a. 2010 a: 17). Mit dieser Materie hatte sich schon Lasswell in „Psychopathology and Politics“ (1930) eingehend beschäftigt. Volkan und Ast (1994) oder auch Wirth $(2002,2007)$ als deutscher Vertreter haben sich neben zahlreichen anderen Autoren der Erforschung des Narzissmus und der narzisstischen Persönlichkeitsstörung gewidmet.

In der kritischen Auseinandersetzung und Weiterentwicklung der psychoanalytischen Ansätze haben sich die Trait- und Motivtheorien besonders hervorgetan. Traittheorien beschäftigen sich mit der Analyse von Persönlichkeitseigenschaften, die unabhängig von Zeitpunkt und Situation als stabil angesehen werden (Cottam u. a. 2010 a: 19). Einer der elaboriertesten Ansätze in diesem Bereich ist der sogenannte „Big Five“-Ansatz, der die Persönlichkeitsausprägung eines Individuums anhand von fünf Faktoren - ,, neuroticism“, , extroversion“, ,, agreeableness", ,,openness to experience" und ,conscientiousness" - bestimmt. Ein aktueller Sammelband der deutschen empirischen Sozialforschung zum Thema „Big Five“ wurde von Schumann und Schoen herausgegeben (2005). Die interdisziplinär zu verortenden Einzelbeiträge erörtern den Zusammenhang von „Big Five“-Persönlichkeitsvariablen und Wahlverhalten, politischer Orientierung und Informationsverarbeitung sowie gesellschaftlichem Wertewandel.

Das Interesse der Motivtheorien richtet sich schließlich im Besonderen auf die Antizipation und Verfolgung gewünschter Ziele von Individuen (Winter 2003: 116). Die größte Aufmerksamkeit in Wissenschaft und Forschung genießen dabei die drei Handlungsmotive ,need for power“ (Machtbedürfnis), ,need for affiliation intimacy“ (Bedürfnis nach Zugehörigkeit) sowie ,need for achievement“ (Bedürfnis nach Leistung) (ebd.: 121; Cottam u. a. 2010 a: 21). Zahlreiche Publikationen haben diese drei Motive an unterschiedlichen politischen Entscheidungsträgern untersucht, so etwa in der vergleichenden Studie zu George Bush sen. und Michail Gorbatschow (Winter u. a. 1991) oder in der Analyse zu Motiven von US-Präsidenten (Winter 1987).

Ein integrativer und in besonderem Maße ausdifferenzierter Ansatz der Persönlichkeitsforschung stellt die Leadership Trait Analysis (LTA) von Hermann dar. Diese kombiniert die verschiedenen Elemente (Eigenschaften, Motivation, Überzeugungen, Kognition etc.) der Persönlichkeit zu einem konsistenten Analyserahmen. Die LTA untersucht den Zusammenhang von Persönlichkeit und Verhalten politischer Führungspersönlichkeiten und beruht auf der in der politischen Ideengeschichte fest verwurzelten Annahme, dass Eigenschaften politischer Führungs- 
persönlichkeiten (Traits) Auswirkungen auf deren politisches Verhalten und somit auch auf deren Führungsstil haben (Hermann 1974: 201 f.). Hermann hat acht Führungsstile (,expansionistic“, „evangelistic“, ,actively independent“, „directive“, ,,incremental“, ,influential“, „opportunistic“ und „,collegial“) definiert, die sowohl Überzeugungen als auch Entscheidungsstil und Handlungsmotivation des Politikers integrieren.

Die LTA bildet einen Analyserahmen, mit dem sieben Führungseigenschaften anhand der spontanen Sprache eines Politikers untersucht werden, ohne mit dem Politiker dabei selbst direkt in Kontakt treten zu müssen (assessment-at-a-distance technique). Diese sieben Traits sind: (1) „belief in one's own ability to control events“, (2) „,need for power and influence“, (3) „,self-confidence“, (4) ,conceptual complexity“, (5) „task focus vs. interpersonal focus“, (6) „distrust of others“, (7) „,ingroup bias“. Aus dem Verhältnis der zu analysierenden Führungseigenschaften zueinander kann schließlich der persönliche Führungsstil ermittelt werden, der Antworten darauf gibt, wie politische Akteure auf Restriktionen in ihrem Umfeld reagieren, auf welcher Informationsgrundlage sie ihre Entscheidungen treffen und welche Motivation sie in ihrer politischen Arbeit antreibt (Hermann/Hermann 1983; Hermann 1987, 2002 a).

Die LTA wurde neben anderen Analysetechniken im Center for the Analysis of Personality and Political Behavior (CAPPB) der CIA zur Erstellung psychologischer Persönlichkeitsprofile hochrangiger ausländischer Politiker angewendet (Post 2005 b: 51 f.). Hermann hat beginnend mit ihrer Zeit am CAPPB mithilfe der LTA zahlreiche vergleichende Studien zu politischen Führungspersönlichkeiten angefertigt, so beispielsweise eine Studie über die außenpolitische Orientierung von zwölf Staatsführern des subsaharischen Afrikas (Hermann/Hermann 1983), eine zu den Mitgliedern des Politbüros der KPdSU (1980 a), eine Studie zu den Führungsstilen britischer Premierminister und deutscher Kanzler (Hermann/Kaarbo 1998) oder Persönlichkeitsprofile zu Bill Clinton und Saddam Hussein (2005 a, 2005 b). Das Verfahren, das insbesondere der Analyse hochrangiger politischer Entscheidungsträger dient, wird indes auch auf einen größeren Personenkreis angewendet. Kille adaptierte die auf Regierungschefs von Nationalstaaten ausgerichtete LTA und untersuchte die Führungsstile ausgewählter UN-Generalsekretäre (2006). Lazarevska u. a. analysierten hingegen das Sprachverhalten von Terroristen sowie dessen Unterschiede zur Sprache nationaler Staats- und Regierungschefs (Lazarevska/ Sholl/Young 2005). Auch einzelne Führungseigenschaften der LTA wurden untersucht, wie zum Beispiel die „,conceptual complexity“ von Zentralbankern im Verlauf der asiatischen Finanzkrise in den späten 1990er Jahren (Thies 2009). 


\section{Perspektiven und Potentiale der Politischen Psychologie}

Die Frage des praktischen Nutzens und der Relevanz begleitet die Politische Psychologie seit ihren Anfängen (McDermott 2004: $10 \mathrm{ff}$.). Worin bestehen der Mehrwert und mögliche Anknüpfungspunkte für die deutschsprachige Politikwissenschaft?

Für Hermann bietet die PP eine alternative Perspektive, politische Akteure und politische Prozesse in ihrer Interaktion zu analysieren (2002 b: 46). Ward hingegen betrachtet den Nutzen der PP kritischer und hebt einen Punkt hervor, der häufig thematisiert wird: die Frage nach ihrer „Objektivität“. In der Tat bleibt die Politische Psychologie von einer Ambivalenz gekennzeichnet, da ihre Erkenntnisse und Konzepte nicht nur zur Aufklärung oder Prävention, sondern auch zur gezielten politischen Manipulation verwendet werden können (2002: 75 f.). Dies anerkennend argumentiert Crenshaw (2002: 142-145) jedoch, dass sich der Nutzen der PP gerade mit Blick auf virulente Fragestellungen erweist. Dies exemplifiziert sie anhand politischer Probleme wie ethnischer Konflikte, Terrorismus oder der Verbreitung von Massenvernichtungswaffen. Gerade ihre interdisziplinäre Ausrichtung ermöglicht, Erkenntnisse zu komplexen politischen Prozessen und Phänomenen aufzuschlüsseln. Dies betont auch Hilgers, der allerdings davor warnt, die PP im Kontext konkreter politischer Probleme, wie beispielsweise im Umgang mit Rechtsextremismus, als Allheilmittel oder gar als „Erlösungsstrategie“ zu betrachten (2007: 6). Vielmehr könne sie im Konfliktfall Dynamiken aufzeigen, Handlungskompetenzen liefern und zur Aufklärung gesellschaftlicher Widersprüche, Interessenskonflikte oder Ungerechtigkeiten beitragen (ebd.).

Auch Deutsch und Kinnvall unterstreichen den gesellschaftlichen Nutzen der PP. Ihnen zufolge können die durch die PP generierten Einblicke und Erkenntnisse zur Verbesserung politischer Prozesse beitragen. Mehr noch: Über die üblichen Handlungsempfehlungen hinaus stellt die PP den politischen Praktikern Rahmenbedingungen, Ideen und systematische Konzepte zur Verfügung, mit Hilfe derer sie ihre jeweiligen Aktivitäten umfassender und in einer analytischen Art und Weise hinterfragen und bewerten können (2002: 19). Obwohl der Zugang zur PP aufgrund des komplexen und breit gefächerten Themen- und Methodenspektrums kein einfacher ist, ermöglicht die Auseinandersetzung mit ihr ein tiefergehendes Verständnis von den Ursachen politischen Verhaltens und anderer politischer Phänomene.

Auch für die deutschsprachige politikwissenschaftliche Analyse kann die PP neue Perspektiven eröffnen und Anknüpfungspunkte identifizieren. Für viele Teilbereiche der Politikwissenschaft lassen sich Bezüge zur PP finden. In der politischen Theorie und Ideengeschichte können die Fragen zur Natur des Menschen, zur po- 
litischen Führung oder zur politisch-gesellschaftlichen Ordnung in Verbindung mit politisch-psychologischen Ansätzen neue Einblicke ermöglichen. Innovative Erkenntnisse der Wahl- und Einstellungsforschung (vgl. Faas/Arzheimer/Roßteutscher 2010) können im Bereich des politischen Systems Deutschlands für die Analyse fruchtbar gemacht werden. Die Analyse außenpolitischer Orientierungen und kognitiver Entscheidungsfindungsfindungsprozesse sowie das Profiling von Staatsführern können sowohl für den Bereich der Internationalen Beziehungen als auch für die Vergleichende Politikwissenschaft gewinnbringende Einsichten liefern. In der Politikdidaktik kann die PP für die Entschlüsselung von politischen Einstellungen und politischem Verhalten sowie für die politische Bildung im Bereich der Demokratieförderung und des zivilgesellschaftlichen Engagements von großer Relevanz sein (Frankenberger/Frech/Grimm 2007). Überall dort, wo der Faktor Persönlichkeit im Spannungsverhältnis von Amt und Person oder individuelle bzw. kollektive Akteure im Spannungsverhältnis von „Agency“ und „Structure“ zum Tragen kommen, kann die Politische Psychologie die Perspektive weiten und den Erkenntnisgewinn vergrößern.

\section{Literatur}

Adorno, Theodor W./Frenkel-Brunswik, Else/Levinson, Daniel J., 1950: The Authoritarian Personality. Studies in Prejudice, New York.

Axelrod, Robert (Hrsg.), 1976: Structure of Decision. The Cognitive Maps of Political Elites, Princeton/New York.

Baum, Steven K., 2008: The Psychology of Genocide. Perpetrators, Bystanders, and Rescuers, Cambridge.

Bleek, Wilhelm, 2001: Geschichte der Politikwissenschaft in Deutschland, München.

Byman, Daniel/Pollack, Kenneth M., 2001: Let Us Now Praise Great Men. Bringing the Statesman Back In, in: International Security 25 (4), 107-146.

Campbell, Angus/Converse, Philip E./Miller, Warren E./Stokes, Donald E., 1960: The American Voter, New York.

Chirot, Daniel/McCauley, Daniel, 2010: Why Not Kill Them All? Logic and Prevention of Mass Political Murder, Princeton/Oxford.

Cottam, Martha L./Dietz-Uhler, Beth/Mastors, Elene/Preston, Thomas, 2010 a: Political Psychology. Introduction and Overview, in: dies., Introduction to Political Psychology, New York/Hove, 1-12 (2. Aufl.).

Cottam, Martha L./Dietz-Uhler, Beth/Mastors, Elene/Preston, Thomas, 2010 b: Introduction to Political Psychology, New York/Hove (2. Aufl.). 
Crenshaw, Martha, 2002: The Utility of Political Psychology, in: Monroe, Kristen R. (Hrsg.), Political Psychology, Mahwah, 141-150.

Crenshaw, Martha, 2004: The Psychology of Political Terrorism, in: John T. Jost/ Jim Sidanus (Hrsg.), Political Psychology, New York/Hove, 411-431.

Crenshaw, Martha, 2007: Explaining Suicide Terrorism. A Review Essay“, in: Security Studies 16 (1), 133-162.

Cunningham,William A./Johnson, Marcia K./Raye, Carol L./Gatenby, J. Chris/Gore, John C./Banaji, Mahzarin R., 2004: Separable Neural Components in the Processing of Black and White Faces, in: Psychological Science 15 (12), 806-813.

De Rivera, Joseph/Páez, Darío, 2007: Emotional Climate, Human Security, and Cultures of Peace, in: Journal of Social Issues 63 (1), 233-253.

Deutsch, Morton/Kinnvall, Catarina, 2002: What Is Political Psychology?, in: Kristen R. Monroe (Hrsg.), Political Psychology, Mahwah, 15-42.

Dolcos, Florin/Iordan, Alexandru D./Dolcos, Sandra, 2011: Neural Correlates of Emotion-Cognition Interactions. A Review of Evidence from Brain Imaging Investigations, in: Journal of Cognitive Psychology 23 (6), 669-694.

Downs, Anthony, 1957: An Economic Theory of Democracy, New York.

Dumont, Muriel/Yzerbyt, Vincent/Wigboldus, Daniël/Gordijn, Ernestine H., 2003:

Social Categorization and Fear Reactions to the September 11th Terrorist Attacks, in: Personality and Social Psychology Bulletin 29 (12), 1509-1520.

Erikson, Erik H., 1958: Young Man Luther. A Study in Psychoanalysis and History, New York.

Faas, Thorsten/Arzheimer, Kai/Roßteutscher, Sigrid (Hrsg.), 2010: Information Wahrnehmung - Emotion, Wiesbaden.

Fagagnini, Hans Peter, 2000: Was soll denn politische Führung?, in: Zeitschrift für Politik 47 (3), 274-292.

Festinger, Leon, 1957: A Theory of Cognitive Dissonance, Evanston.

Fliegauf, Mark T./Kießling, Andreas/Novy, Leonard, 2008: Leader und Follower Grundzüge eines inter-personalen Ansatzes zur Analyse politischer Führungsleistung, in: Zeitschrift für Politikwissenschaft 18 (4), S. 399-421.

Frankenberger, Rolf/Frech, Siegfried/Grimm, Daniela (Hrsg.), 2007: Politische Psychologie und politische Bildung, Schwalbach/Ts.

Frick, Lothar, 2007: Vorwort, in: Rolf Frankenberger/Siegfried Frech/Daniela Grimm (Hrsg.), Politische Psychologie und politische Bildung, Schwalbach/Ts., 9-11.

Gabriel, Oscar W./Wessels, Bernhard/Falter, Jürgen W. (Hrsg.), 2009: Wahlen und Wähler. Analysen aus Anlass der Bundestagswahl 2005, Wiesbaden. 
Politische Psychologie

Gast, Henrik, 2007: Führung durch Kommunikation. Eine Übersicht über die Handlungsressourcen des Bundeskanzlers, in: Werner J. Patzelt/Martin Sebaldt/Uwe Kranenpohl (Hrsg.), Res publica semper reformanda. Wissenschaft und politische Bildung im Dienste des Gemeinwohls, Wiesbaden, 268-290.

Gast, Henrik, 2009: Was bedeutet ,politische Führung“? Eine Übersicht über unterschiedliche Definitionen aus interdisziplinärer Perspektive und ein Vorschlag zur systematischen Analyse des Gegenstandes anhand des Strukturfunktionalismus, in: Zeitschrift für Politikberatung 2 (2), 211-229.

Geise, Stephanie/Brettschneider, Frank, 2010: Die Wahrnehmung und Bewertung von Wahlplakaten. Ergebnisse einer Eyetracking-Studie, in: Thorsten Faas/Kai Arzheimer/Sigrid Roßteutscher (Hrsg.), Information - Wahrnehmung - Emotion, Wiesbaden, 71-95.

George, Alexander L., 1971: The "Operational Code". A Neglected Approach to the Study of Political Leaders and Decision-Making, in: Erik P. Hoffmann/Frederic J. Fleron Jr. (Hrsg.), The Conduct of Soviet Foreign Policy, Chicago/New York, 165-190.

George, Alexander L./George, Juliette L., 1956: Woodrow Wilson and Colonel House. A Personality Study, New York.

Glaab, Manuela, 2010: Political Leadership in der Großen Koalition. Führungsressourcen und -stile von Bundeskanzlerin Merkel, in: Christoph Egle/Reimut Zohlnhöfer (Hrsg.), Die zweite Große Koalition. Eine Bilanz der Regierung Merkel 2005-2009, Wiesbaden, 123-155.

Grasselt, Nico/Korte, Karl-Rudolf, 2007: Führung in Politik und Wirtschaft. Instrumente, Stile und Techniken, Wiesbaden.

Greenstein, Fred Irwin, 1969: Personality and Politics. Problems of Evidence, Inference, and Conceptualization, Chicago.

Greenstein, Fred Irwin, 1992: Can Personality and Politics Be Studied Systematically?, in: Political Psychology 13 (1), 105-128.

Gross Stein, Janice, 2008: Psychological Explanation of International Conflict, in:

Walter Carlsnaes (Hrsg.), Handbook of International Relations, London, 292-308.

Hartmann, Jürgen, 2007: Persönlichkeit und Politik, Wiesbaden.

Healy, Alice F./Aylward, Alison G./Bourne Jr., Lyle E../Beer, Francis A., 2009:

Terrorism After 9/11. Reactions to Simulated News Reports, in: The American Journal of Psychology 122 (2), 153-165.

Helms, Ludger, 2000: „Politische Führung“ als politikwissenschaftliches Problem, in: Politische Vierteljahresschrift 41 (3), 411-434. 
Helms, Ludger, 2005: Regierungsorganisation und politische Führung in Deutschland, Wiesbaden.

Hermann, Margaret G., 1974: Leader Personality and Foreign Policy Behavior, in: James N. Rosenau (Hrsg.), Comparing Foreign Policies. Theories, Findings and Methods, New York, 201-234.

Hermann, Margaret G., 1980 a: Assessing the Personalities of Soviet Politburo Members, in: Personality and Social Psychology Bulletin 6 (3), 332-352.

Hermann, Margaret G., 1980 b: Explaining Foreign Policy Behavior Using the Personal Characteristics of Political Leaders, in: International Studies Quarterly 24 (1), 7-46.

Hermann, Margaret G., 1986: What Is Political Psychology?, in: dies. (Hrsg.), Political Psychology. Contemporary Problems and Issues, San Francisco, 1-10.

Hermann, Margaret G., 1987: Handbook for Assessing Personal Characteristics and Foreign Policy Orientation of Political Leaders, Mershon Center, Ohio State University.

Hermann, Margaret G., 1993: Leaders and Foreign Policy Decision-making, in: Dan Caldwell/Timothy J. McKeown (Hrsg.), Diplomacy, Force, and Leadership. Essays in Honor of Alexander L. George, Boulder u. a., 77-94.

Hermann, Margaret G., 2001: How Decision Units Shape Foreign Policy: A Theoretical Framework, in: International Studies Review 3 (2), Leaders, Groups, and Coalitions: Understanding the People and Processes in Foreign Policymaking, 47-81.

Hermann, Margaret G., 2002 a: Assessing Leadership Style: A Trait Analysis, Hilliard.

Hermann, Margaret G., 2002 b: Political Psychology as a Perspective in the Study of Politics, in: Kristen R. Monroe (Hrsg.), Political Psychology, Mahwah, 43-61.

Hermann, Margaret G., 2004 a: Preface: Political Psychology as a Perspective on Politics, in: dies. (Hrsg.), Advances in Political Psychology 1, Amsterdam u. a., 11-19.

Hermann, Margaret G. (Hrsg.), 2004 b: Advances in Political Psychology 1, Amsterdam u. a.

Hermann, Margaret G., 2005 a: William Jefferson Clinton's Leadership Style, in: Jerrold M. Post (Hrsg.), The Psychological Assessment of Political Leaders: With Profiles of Saddam Hussein and Bill Clinton, Ann Arbor, 313-323 (2. Aufl.).

Hermann, Margaret G., 2005 b: Saddam Hussein's Leadership Style, in: Jerrold M. Post (Hrsg.), The Psychological Assessment of Political Leaders: With Profiles of Saddam Hussein and Bill Clinton, Ann Arbor, 375-386 (2. Aufl.). 
Politische Psychologie

Hermann, Margaret G./Hagan, Joe D., 1998: International Decision Making: Leadership Matters, in: Foreign Policy 110, Special Edition: Frontiers of Knowledge, 124-137.

Hermann, Margaret G./Hermann, Charles F., 1983: Factors of Leadership in SubSaharan Africa: Possible Determinants of Foreign Policy Behavior (Report prepared for the Defense Intelligence Agency of the Department of Defense under Contract MDA908-82-C-0191), Mershon Center, Ohio State University.

Hermann, Margaret G./Kaarbo, Juliet, 1998: Leadership Styles of Prime Ministers. How Individual Differences Affect The Foreign Policymaking Process, in: The Leadership Quarterly 9 (3), 243-263.

Hilgers, Micha, 2007: Psychologistischer Firlefanz oder Teil innovativer Politik?, in: APuZ 11, 3-6.

Houghton, David Patrick, 2009: Political Psychology. Situations, Individuals, and Cases, New York/London.

Jankowitsch, Regina M., 2008: Political Leadership. Ein Vergleich der Entwicklungen in den USA, Deutschland und Österreich, in: Johannes Pollack/Fritz Sager/Ulrich Sarcinelli/Annette Zimmer (Hrsg.), Politik und Persönlichkeit, Wien, 197-210.

Johanson, Aki/Gustafson, Lars/Passant, Ulla/Risberg, Jarl/Smith, Gudmund/Warkentin, Siegbert/Tucker, Don, 1998: Brain Function in Spider Phobia, in: Psychiatry Research 84 (2/3), 101-111.

Jones, David/Kanhouse, Edward/Kelley, Harold/Nisbett, Richard/Valins, Stuart/ Weiner, Berhard (Hrsg.), 1972: Attribution. Perceiving the Causes of Behavior, Morristown.

Jost, John T./Sidanius, Jim, 2004 a: Political Psychology. An Introduction, in: dies. (Hrsg.), Political Psychology. Key Readings, New York/Howe, 1-17.

Jost, John T./Sidanius, Jim (Hrsg.), 2004 b: Political Psychology. Key Readings, New York/Howe.

Kaplan, Jonas T./Freedman, Joshua/Iacoboni, Marco, 2007: Us Versus Them. Political Attitudes and Party Affiliation Influence Neural Response to Faces of Presidential Candidates, in: Neuropsychologia 45 (1), 55-64.

Kille, Kent J., 2006: From Manger to Visionary. The Secretary-General of the United Nations, New York u. a.

Knutson, Jeanne N. (Hrsg.), 1973: Handbook of Political Psychology, San Francisco.

Kuklinski, James/Luskin, Robert/Bolland, John, 1991: Where's the Schema? Going Beyond the ,S' Word in Political Psychology, in: American Political Science Review 85, 1341-1356. 
Lasswell, Harold D., 1930: Psychopathology and Politics, New York.

Lasswell, Harold D., 1935: World Politics and Personal Insecurity, New York.

Lasswell, Harold D., 1936: Politics: Who Gets What, When and How, New York.

Lasswell, Harold D., 1948: Power and Personality, Westport.

Lazarevska, Elena/Sholl, Jayne M./Young, Michael, 2005: The Distinctive Language of Terrorists (Prepared for Presentation at the 46th Annual Convention of the International Studies Association, March 1-5, Honolulu).

Leach, Colin Wayne, 2010: The Person in Political Emotion, in: Journal of Personality 78 (6), 1827-1859.

Mandel, Robert, 1986: Psychological Approaches to International Relations, in: Hermann, Margaret G. (Hrsg.), Political Psychology: Contemporary Problems and Issues, San Francisco, 251-278.

Mayer, Tilman/Kortmann, Martina, 2010: Politische Kultur, in: Irene Gerlach/Eckhard Jesse/Marianne Kneuer/Nikolaus Werz (Hrsg.), 2010: Politikwissenschaft in Deutschland, Baden-Baden.

McDermott, Rose, 2004: Political Psychology in International Relations, Ann Arbor.

McGuire, William J., 2004: The Poly-Psy Relationship. Three Phases of a Long Affair, in: John T. Jost/Jim Sidanius (Hrsg.), Political Psychology, New York/ Howe, 23-31.

Monroe, Kristen R., 2002 a: Political Psychology: An Overview, in: dies. (Hrsg.), Political Psychology, Mahwah, 1-11.

Monroe, Kristen R. (Hrsg.), 2002 b: Political Psychology, Mahwah.

Montada, Leo/Schneider, Angela, 1989: Justice and Emotional Reactions to the Disadvantaged, in: Social Justice Research 3 (4), 313-344.

Pickel, Susanne/Pickel Gert, 2006: Politische Kultur und Demokratieforschung. Grundbegriffe, Theorien, Methoden, Wiesbaden.

Post, Jerrold M., 2005 a: When Hatred is Bred in the Bone: Psycho-Cultural Foundations of Contemporary Terrorism, in: Political Psychology 26 (4), Malden, 615-636.

Post, Jerrold M., 2005 b: Leader Personality Assessments in Support of Government Policy, in: ders. (Hrsg.), The Psychological Assessment of Political Leaders: With Profiles of Saddam Hussein and Bill Clinton, Ann Arbor, 39-61 (2. Aufl.). Post, Jerrold M., 2009: The Mind of the Terrorist. The Psychology of Terrorism from the IRA to Al-Qaeda, New York.

Post, Jerrold M./Walker, Stephen G./Winter, David G., 2005: Profiling Political Leaders. An Introduction, in: Jerrold M. Post (Hrsg.), The Psychological As- 
Politische Psychologie

sessment of Political Leaders: With Profiles of Saddam Hussein and Bill Clinton, Ann Arbor, 1-7 (2. Aufl.).

Preiser, Siegfried, 1993: Politische Psychologie, in: Angela Schorr (Hrsg.), Handwörterbuch der Angewandten Psychologie. Die Angewandte Psychologie in Schlüsselbegriffen, Bonn.

Renshon, Jonathan/Renshon, Stanley A., 2008: The Theory and Practice of Foreign Policy Decision Making, in: Political Psychology 29 (4), 509-536.

Richeson, Jennifer A./Baird, Abigail A./Gordon, Heather L./Heatherton, Todd F./ Wyland, Carrie L./Trawalter, Sophie, J./Shelton, Nicole, 2003: An fMRI Investigation of the Impact of Interracial Contact on Executive Function, in: Nature Neuroscience 6 (12), 1323-1328.

Schemer, Christian/Wirth, Werner/Matthes, Jörg, 2010: Kognitive und affektive Einflüsse auf Einstellungen in direktdemokratischen Kampagnen, in: Thorsten Faas/Kai Arzheimer/Sigrid Roßteutscher (Hrsg.), 2010: Information - Wahrnehmung - Emotion, Wiesbaden, 277-289.

Schumann, Siegfried, 2005 (unter Mitarbeit von Harald Schoen): Persönlichkeit.

Eine vergessene Größe der empirischen Sozialforschung, Wiesbaden.

Sears, David O./Huddy, Leonie/Jervis, Robert, 2003 a: The Psychologies Underlying Political Psychology, in: dies. (Hrsg.), Oxford Handbook of Political Psychology, Oxford/New York, 3-16.

Sears, David O./Huddy, Leonie/Jervis, Robert (Hrsg.), 2003 b: Oxford Handbook of Political Psychology, Oxford/New York.

Sebaldt, Martin/Gast, Henrik (Hrsg.), 2010: Politische Führung in westlichen Regierungssystemen. Theorie und Praxis im internationalen Vergleich, Wiesbaden. Skitka, Linda J., 2002: Do the Means Always Justify the Ends, or Do the Ends Sometimes Justify the Means? A Value Protection Model of Justice Reasoning, in: Personality and Social Psychology Bulletin 28 (5), 588-597.

Staub, Ervin, 2000: Genocide and Mass Killings. Origins, Prevention, Healing and Reconciliation, in: Political Psychology 21 (2), 367-382.

Staub, Ervin, 2006: Reconciliation after Genocide, Mass Killing, or Intractable Conflict: Understanding the Roots of Violence, Psychological Recovery, and Steps toward a General Theory, in: Political Psychology 27 (6), 867-894.

Thies, Cameron G., 2009: The Conceptual Complexity of Central Bankers and the Asian Financial Crisis, in: Political Psychology 30 (3), 445-464.

Volkan, Vamik D., 1999: Das Versagen der Diplomatie. Zur Psychoanalyse nationaler, ethnischer und religiöser Konflikte, Gießen.

Volkan, Vamik D./Ast, Gabriele, 1994: Spektrum des Narzissmus. Eine klinische Studie des gesunden Narzissmus, des narzisstisch-masochistischen Charakters, 
der narzisstischen Persönlichkeitsorganisation, des malignen Narzissmus und des erfolgreichen Narzissmus, Göttingen.

Walker, Iain/Smith, Heather J., 2002: Relative Deprivation. Specification, Development, and Integration, Cambridge.

Ward, Dana, 2002: Political Psychology: Origins and Development, in: Kristen R.

Monroe (Hrsg.), Political Psychology, Mahwah, 61-79.

Wasmuth, Ulrike C., 2011: Politische Psychologie, in: Dieter Nohlen/Florian Grotz (Hrsg.), Kleines Lexikon der Politik, München, 471 (5. Aufl.).

Westen, Drew/Blagov, Pavel S./Harenski, Keith/Kilts, Clint/Hamann, Stephan, 2006: Neural Bases of Motivated Reasoning. An fMRI Study of Emotional Constraints on Partisan Political Judgment in the 2004 U.S. Presidential Election, in: Journal Cognitive Neuroscience 18 (11), 1947-1958.

Westle, Bettina/Gabriel, Oscar W. (Hrsg.), 2009: Politische Kultur. Eine Einführung, Baden-Baden.

Winter, David G., 1987: Leader Appeal, Leader Performance, and the Motive Profiles of Leaders and Followers: A Study of American Presidents and Elections, in: Journal of Personality and Social Psychology 52 (1), 196-202.

Winter, David G., 1992: Personality and Foreign Policy Behavior. Historical Overview of Research, in: Eric Singer/Valerie Hudson (Hrsg.), Political Psychology and Foreign Policy, Boulder u. a., 79-101.

Winter, David G., 2003: Personality and Political Behavior, in: David O. Sears/ Leonie Huddy/Robert Jervis (Hrsg.), Oxford Handbook of Political Psychology, Oxford/New York, 110-144.

Winter, David G., 2005: Assessing Leaders' Personalities: A Historical Survey of Academic Research Studies, in: Jerrold M. Post (Hrsg.), The Psychological Assessment of Political Leaders: With Profiles of Saddam Hussein and Bill Clinton, Ann Arbor, 11-38 (2. Aufl.).

Winter, David G./Hermann, Margaret G./Weintraub, Walter/Walker, Stephen G., 1991: The Personalities of Bush and Gorbachev Measured at a Distance. Procedures, Portraits and Policy, in: Political Psychology 12, 215-245.

Wirth, Hans-Jürgen, 2002: Narzissmus und Macht. Zur Psychoanalyse seelischer Störungen in der Politik, Gießen.

Wirth, Hans-Jürgen, 2007: Macht, Narzissmus und die Sehnsucht nach dem Führer, in: APuZ 11, 13-18.

Zamboni, Giovanna/Gozzi, Marta/Krueger, Frank/Duhamel, Jean-René/Sirigu, Angela/Grafman, Jordan, 2009: Individualism, Conservatism, and Radicalism as Criteria for Processing Political Beliefs. A Parametric fMRI Study, in: Journal Social Neuroscience 4 (5), 367-383. 
Korrespondenzanschrift:

Dorothea Prell (M.A.)

Institut für Politikwissenschaft

Friedrich-Schiller-Universität Jena

Carl-Zeiß-Str. 3

07743 Jena

E-Mail: dorothea.prell@uni-jena.de

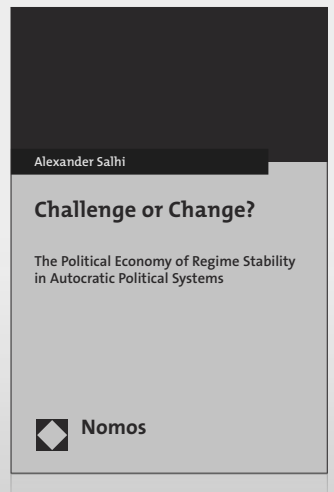

\section{Challenge or Change?}

The Political Economy of Regime Stability

in Autocratic Political Systems

Von Alexander Salhi

2012, 120 S., brosch., 24,- $€$

ISBN 978-3-8329-7016-1

Die Beziehung der Hauptinstrumente autokratischer Herrschaft, nämlich Repression und ökonomische Wohlfahrt, wird aus Sicht der politischen Ökonomie modelltheoretisch neu betrachtet. Als entscheidender Faktor für die langfristige Regimestabilität wird dabei eine Substitutionsbeziehung der beiden Instrumente identifiziert.

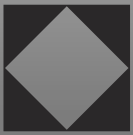

